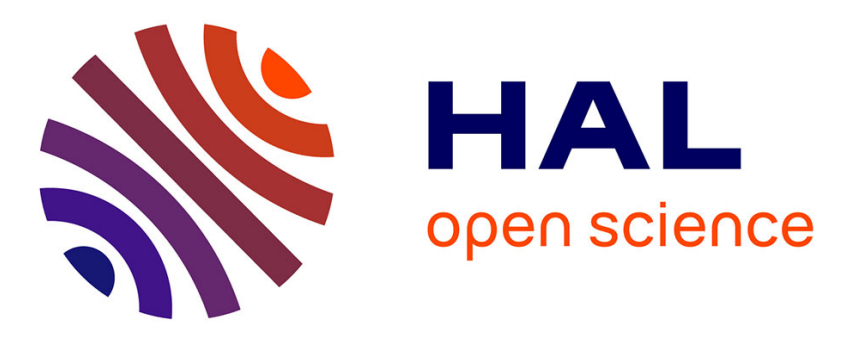

\title{
Determination of reaction parameters for cardboard thermal degradation using experimental data
}

\author{
T Loulou, Sylvain Salvador, Jean-Louis Dirion
}

\section{To cite this version:}

T Loulou, Sylvain Salvador, Jean-Louis Dirion. Determination of reaction parameters for cardboard thermal degradation using experimental data. Chemical Engineering Research and Design, 2003, 81 (A9), pp.1265-1270. 10.1205/026387603770866489 . hal-01718082

\section{HAL Id: hal-01718082 \\ https://hal.science/hal-01718082}

Submitted on 1 Jun 2018

HAL is a multi-disciplinary open access archive for the deposit and dissemination of scientific research documents, whether they are published or not. The documents may come from teaching and research institutions in France or abroad, or from public or private research centers.
L'archive ouverte pluridisciplinaire HAL, est destinée au dépôt et à la diffusion de documents scientifiques de niveau recherche, publiés ou non, émanant des établissements d'enseignement et de recherche français ou étrangers, des laboratoires publics ou privés. 


\title{
DETERMINATION OF REACTION PARAMETERS FOR CARDBOARD THERMAL DEGRADATION USING EXPERIMENTAL DATA
}

\author{
T. LOULOU, S. SALVADOR and J. L. DIRION \\ Ecole des Mines d'Albi-Carmaux, Laboratoire de Gnie des Procds des solides diviss, UMR CNRS 2393 Albi, France
}

$\mathrm{T}$

his work is part of an ongoing research effort in which parameter estimation techniques have been utilized in recovering the kinetic parameters of a given reacting model using measurements collected from TGA devices. The goal of this research work is to develop a useful and universal estimation procedure to determine simultaneously the kinetic parameters involved in the chemical modelling under study such as combustion, pyrolysis, waste stabilization, etc. The present parameter estimation problem is solved with the Levenberg-Marquardt method of minimization of the least square norm representing the square difference between the measured mass variations during cardboard pyrolysis and the mass responses obtained with numerical solution of the model. An analysis of the linear dependency of the pyrolysis parameters needed to design a robust estimation tool is presented. In order to perform this analysis, the sensitivity coefficients and the sensitivity matrix determinant were examined. Experimental data obtained with TGA during the pyrolysis process of cardboard are analysed in this paper and the unknown parameters involved in the kinetic modelling are estimated.

Keywords: parameter estimation; inverse problem; sensitivity coefficient; kinetic model.

\section{INTRODUCTION}

In building the reaction schemes for solids decomposition, the estimation of kinetic parameters represents a crucial step in chemical modelling (Font et al., 2001). Indeed, once a kinetic scheme has been established, two main methods can be used to estimate the corresponding Arrhenius parameters: a graphical method and numerical fitting. The first method is usually limited to a global (single) reaction. When the reacting scheme involves more than one reaction, the graphic method is not adequate and the numerical fitting represents the only alternative. However, the second method needs to be implemented with precautions and efficiency to avoid the determination of physically unacceptable reaction schemes.

In a recent paper (David et al., 2003), the authors presented the determination of a reacting scheme of thermal degradation of cardboard. As mentioned in many references, they have underlined some difficulties in estimating the kinetic parameters involved in the reaction schemes. In the present study the authors present a numerical tool developed to alleviate the estimation procedure in terms of the computing time and simplicity of use. Physical and chemical considerations, such as positiveness of kinetic parameter, continuity in reaction rate values, are also taken into consideration.

The literature is rich in this field and one can find several commercial and educational softwares such as Themokinetics (Opfermann, 2000), Imsl (IMSL, 1987), AKTS-TA
(Roduit \& Baiker, 1996) which help in the solution of such difficult parameter estimation problems. In this sense, the presented algorithm does not present any originality with respect to the existing software, except for the detailed sensitivity analysis shown in the next section and the incorporation of the constraints on the parameters to be estimated. In fact the sensitivity analysis plays an important part in understanding the effect of each parameter present in the kinetic schemes developed here.

This work is part of an ongoing research effort to develop a practical, universal and minimally time-consuming computing tool that will be helpful in the determination of kinetic parameters occurring in the modelling of any chemical and/or physical problem such as combustion, pyrolysis, stabilization and other phenomena investigated in our laboratory. The estimation of unknowns is formulated as an inverse problem of simultaneously estimating the kinetic parameters involved in the modelling of the physical problem under investigation.

This paper presents an application of this estimation tool with data obtained, during pyrolysis of cardboard, by a thermal gravimetric analysis device (TGA). The minimization of the least square norm is achieved using the Levenberg-Marquardt method (Levenberg, 1944; Marquardt, 1963; More, 1978; Bard, 1974). In order to investigate the estimation feasibility, the sensitivity coefficients and the sensitivity matrix determinant are examined. This analysis helps in quantifying the correlation degree among the parameters of interest. 


\section{DIRECT PROBLEM}

In a previous paper (David et al., 2003), the authors demonstrated that the pyrolysis of cardboard can be described with a two steps reaction scheme, i.e.

$$
\text { Cardboard } \stackrel{F 1}{\longrightarrow} \text { Intermediate } \stackrel{F 1}{\longrightarrow} \text { Char }
$$

In a first reaction, the initial cardboard mass $m_{1}(t)$, is converted into an intermediate pseudo-species denoted $m_{2}(t)$ and gas. Then, the pseudo-species is converted into char, denoted $m_{3}(t)$, and gas through a second reaction. This scheme was chosen from eight proposed kinetic schemes (David et al., 2003). The best fitting of experimental data recorded with three different heating rates was obtained with this scheme. At a given time $t$, the total mass of the sample is the sum of the masses of cardboard, intermediate pseudospecies and char, i.e.

$$
M(t)=m_{1}(t)+m_{2}(t)+m_{3}(t)
$$

The mathematical model, describing the time evolution of the different mass fractions during the pyrolysis process, is based on set of first-order differential equations. The weight loss phenomenon of the cardboard pyrolysis is described by:

$$
\begin{aligned}
& \frac{\mathrm{d} m_{1}}{\mathrm{~d} t}=-k_{1} m_{1} \\
& \frac{\mathrm{d} m_{2}}{\mathrm{~d} t}=+a\left[k_{1} m_{1}-k_{2} m_{2}\right] \\
& \frac{\mathrm{d} m_{3}}{\mathrm{~d} t}=+b k_{2} m_{2}
\end{aligned}
$$

with the following initial boundary conditions

$$
m_{1}(0)=1 . \quad m_{2}(0)=0 . \quad m_{3}(0)=0
$$

The decomposition rule gives:

$$
\frac{\mathrm{d} m_{i}}{\mathrm{~d} t}=\frac{\mathrm{d} m_{i}}{\mathrm{~d} T} \times \frac{\mathrm{d} T}{\mathrm{~d} t}=\frac{\mathrm{d} m_{i}}{\mathrm{~d} T} \beta \quad i=1,2,3
$$

where $\beta$ represents the heating rate, which is considered constant in this study. The kinetic reaction rates depend on the absolute temperature and are given by the two Arrhenius functions:

$$
k_{i}=A_{i} \exp \left[-\frac{E_{i}}{R T}\right] \quad i=1,2
$$

where $A_{i}$ is the Arrhenius factor, $E_{i}$ is the activation energy for the pyrolysis, $R$ is the ideal gas constant, and $T$ is the absolute temperature of the reaction. $a$ and $b$ represent the mass stoichiometric coefficients.

In the direct problem associated with the kinetic reaction described above, the kinetic parameters $A_{1}, E_{1}, A_{2}, E_{2}$, as well as the coefficients $a, b$, initial boundary conditions, are known. The objective of the direct problem is then to determine the time variation of mass fractions, $m_{i}(t), i=1,2,3$, and the total mass $M(t)$ during the pyrolysis process.

There exists no analytical solution for the direct problem given in equations (2)-(5). As we are dealing with more than one first-order differential equation, the possibility of stiffness of the set of equations can arise. For these two reasons the numerical solution of the direct problem is obtained with the Kaps-Rentrop algorithm in terms of the subroutine stiff developed in Press, et al. (1992).

\section{INVERSE PROBLEM}

Once the kinetic scheme has been established, two principal methods are usually used to estimate the corresponding Arrhenius parameters: the graphical method or numerical fitting. The first method is limited only to single reaction (global). The case of simultaneous reactions is treated through a numerical procedure formulated as an inverse problem.

For the inverse problem considered here, the parameters $A_{1}, E_{1}, A_{2}, E_{2}, a$, and $b$ are regarded as six unknowns, while the other quantities appearing in the formulation of the direct problem described above are assumed to be known precisely. Thus, the vector of the unknown parameters is

$$
\mathbf{U}^{\mathrm{T}}=\left[A_{1}, E_{1}, A_{2}, E_{2}, a, b\right]=\left[u_{1}, u_{2}, u_{3}, u_{4}, u_{5}, u_{6}\right]
$$

The additional information needed in the simultaneously estimation of the kinetic parameters is available from the experimental data obtained with the TGA apparatus.

Generally, inverse problems are solved by minimizing a residual functional $J$ based on the ordinary least square norm. The sum of the squared residuals between the measured data and the responses of a model simulating the physical problem under investigation defines the least square norm. For discrete measured data, the residual functional is written as follows:

$$
J(\mathbf{U})=\sum_{i=1}^{N}\left[Y\left(t_{i}\right)-M\left(t_{i}\right)\right]^{2}
$$

where $M\left(t_{i}\right)$ and $Y\left(t_{i}\right)$ are, respectively, the computed and measured total mass of chart at time $t_{i}$. $N$ is the total number of measurements. In vectorial form, the above expression can be written as

$$
J(\mathbf{U})=[\mathbf{Y}-\mathbf{M}(\mathbf{U})]^{\mathrm{T}}[\mathbf{Y}-\mathbf{M}(\mathbf{U})]
$$

Here, $\mathbf{Y}^{\mathrm{T}}=\left[Y_{1}, Y_{2}, \ldots, Y_{N}\right]$ is the vector of measured mass, $\mathbf{M}^{\mathrm{T}}(\mathbf{U})=\left[M_{1}(\mathbf{U}), M_{2}(\mathbf{U}), \ldots, M_{N}(\mathbf{U})\right]$ is the vector of estimated total mass at time $t_{i},(i=1,2, \ldots, N)$ obtained from the solution of the direct problem with an estimate of vector $\mathbf{U}, \mathbf{U}^{\mathrm{T}}=\left[u_{1}, u_{2}, \ldots, u_{P}\right]$ is the vector of unknowns parameters, $N$ is the total number of measurements, and $P$ is the number of unknown parameters, which is equal to 6 in this case.

A version of Levenberg-Marquardt method was applied for the solution of the presented parameter estimation problem. This method is quite stable, powerful and straightforward and has been applied to a variety of inverse problems. This method belongs to a general class of damped least square methods. The solution for vector $\mathbf{U}$ is achieved using the following iterative procedure:

$$
\begin{aligned}
\mathbf{U}^{(k+1)}= & \mathbf{U}^{(k)}+\left[\left(\mathbf{X}^{(k)}\right)^{\mathrm{T}} \mathbf{X}^{(k)}+\mu^{(k)} \mathbf{\Omega}^{(k)}\right]^{-1} \\
& \times\left(\mathbf{X}^{(k)}\right)^{\mathrm{T}}\left[\mathbf{Y}-\mathbf{M}\left(\mathbf{U}^{(k)}\right)\right]
\end{aligned}
$$


where the superscript $(k)$ defines the iteration number and $\mathbf{X}$ represents the sensitivity matrix evaluated at the iteration $(k)$. The sensitivity matrix is given by:

$$
\mathbf{X}=\left[\frac{\partial \mathbf{M}^{\mathrm{T}}(\mathbf{U})}{\partial \mathbf{U}}\right]^{\mathrm{T}}=\left[\begin{array}{ccc}
\frac{\partial M_{1}}{\partial u_{1}} & \cdots & \frac{\partial M_{1}}{\partial u_{m}} \\
\vdots & \ddots & \vdots \\
\frac{\partial M_{N}}{\partial u_{1}} & \cdots & \frac{\partial M_{N}}{\partial u_{m}}
\end{array}\right]
$$

The elements of the sensitivity matrix $\mathbf{X}$, denoted $X_{i j}$, are known as the sensitivity coefficients. They can provide considerable insight to the estimation problem and in the design of the experiment for optimum accuracy in the estimates.

An iterative procedure is required due to the non-linear nature of the estimation problem because the coefficients of the sensitivity matrix depend on the unknown thermophysical properties to be recovered. Iteration continues until convergence of the estimated parameter is reached, i.e. when there is negligible change in any component of $\mathbf{U}$. One criterion to indicate this is defined as:

$$
\frac{\left|\mathbf{U}^{(k+1)}-\mathbf{U}^{(k)}\right|}{\left|\mathbf{U}^{(k)}\right|} \leqslant \varepsilon
$$

where $\varepsilon$ is a small number to quantify convergence, such as $10^{-5}$. Different versions of the Levenberg-Marquardt method can be found in the literature, depending on the choice of the diagonal of the damping matrix $\Omega^{(k)}$ and of the form chosen for the variation of the damping parameter $\mu^{(k)}$. The most used forms of matrix $\Omega^{(k)}$ are:

$$
\Omega^{(k)}=\mathbf{I} \quad \text { and } \quad \Omega^{(k)}=\operatorname{diag}\left[\left(\mathbf{X}^{(k)}\right)^{\mathrm{T}} \mathbf{X}^{(k)}\right]
$$

where $\mathbf{I}$ is the identity matrix.

\section{RESULTS}

In this section, we present the results obtained with the developed algorithm as applied to the solution of our inverse problem. In the first part of this section, we present a detailed sensitivity analysis to show the feasibility of the estimation. The second part will be dedicated to a numerical test case with its statistical analysis. In the final part we present the kinetic parameters issued from the use experimental data obtained with the pyrolysis of char.

\section{Sensitivity Analysis}

There are several different approaches for the computation of the sensitivity coefficients (Beck and Arnold, 1977). In the present inverse problem the central finite-difference approximation is used to calculate the sensitivity coefficients in the form:

$$
X_{i j}=\frac{M\left(t_{i}, u_{1}, \ldots, u_{j}+\varepsilon u_{j}, \ldots, u_{6}\right)}{-M\left(t_{i}, u_{1}, \ldots, u_{j}-\varepsilon u_{j}, \ldots, u_{6}\right)}
$$

where $\varepsilon=10^{-5}$. The sensitivity coefficient $X_{i j}$, as defined in the previous equation, and in the sensitivity matrix definition given in equation (12), is the measure of the sensitivity of the estimated $M_{i}$ with respect to changes in the parameter $u_{j}$. A small value of the magnitude of $X_{i j}$ indicates that large changes in $u_{j}$ yield small changes in $M_{i}$. The estimation of the parameter $u_{j}$ is extremely difficult in such a case, because basically the same value for total mass would be obtained for a wide range of values of $u_{j}$.

In fact when the sensitivity coefficients are small, we have $\left|\mathbf{X}^{\mathrm{T}} \mathbf{X}\right| \approx 0$ and the inverse problem is ill-conditioned. It can also be shown that $\left|\mathbf{X}^{\mathrm{T}} \mathbf{X}\right|$ is null if any column of $\mathbf{X}$ can expressed as a linear combination of the other columns (Beck and Arnold, 1977). Therefore, it is desirable to have linearly independent sensitivity coefficients $X_{i j}$ with large magnitudes, so that the inverse problem is not very sensitive to measurement errors and accurate estimates of the parameters can be obtained.

Since the unknown parameters can assume different values, the analysis of the sensitivity coefficients is much simplified by using their relative versions defined as:

$$
X_{i j}^{+}=u_{j} \frac{\partial M_{i}}{\partial u_{j}} \quad j=1, \ldots, P
$$

Figure 1 presents the transient behavior of the relative sensitivity coefficients for the six components of $\mathbf{U}$. The exact values of the parameters, used in the sensitivity study, are reported in Table 1. Except for the parameter $E_{1}$, the relative sensitivity coefficients of the remainder parameters are approximately of the same order of magnitude. The smallest relative sensitivity coefficient is observed with the coefficient $A_{2}$, which can have major difficulties in its estimation and a relatively high estimation error. As displayed in Figure 1, the sensitivity coefficients looks slightly linearly dependent, but a careful examination of the different ratios between all the relative coefficients shows that they are not linearly dependent and therefore their simultaneously estimation is feasible. Finally, the analysis of the temperature variation of the determinant $\Delta$ of the matrix $\mathbf{X}^{\mathrm{T}} \mathbf{X}$ reveals that the simultaneous estimation is feasible when the final temperature $T_{\max }$ is equal or greater than $850 \mathrm{~K}$. Indeed, the maximum value of $\Delta$ is reached at $T=850 \mathrm{~K}$.

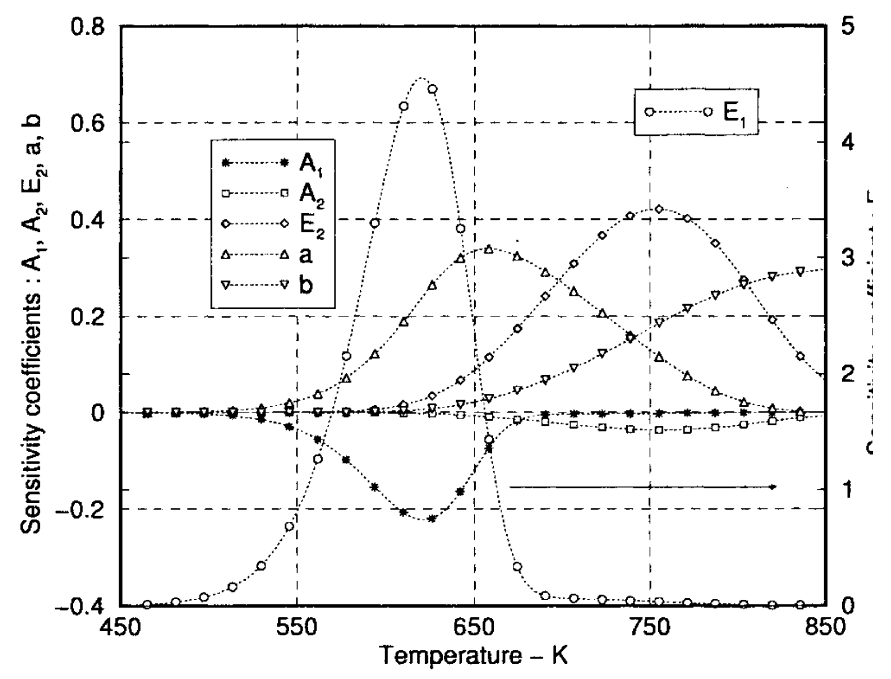

Figure 1. Evolution of relative sensitivity coefficients as temperature function $(T=\beta t)$. 
Table 1. Exact, and estimated parameters and their respective estimation error for two simulated test cases.

\begin{tabular}{|c|c|c|c|c|c|c|}
\hline$\sigma$ & Parameter & Unit & Exact & $\begin{array}{c}\text { Initial } \\
\text { guess }\end{array}$ & Estimated & $\varepsilon_{i}(\%)$ \\
\hline \multirow[t]{6}{*}{0} & $A_{1}$ & $\min ^{-1}$ & $1.07 \times 10^{8}$ & $1.7 \times 10^{6}$ & $1.07 \times 10^{8}$ & 0.0 \\
\hline & $E_{1}$ & $\mathrm{~J} \mathrm{~mol}^{-1}$ & 101,000 & 70,000 & 101,000 & 0.0 \\
\hline & $A_{2}$ & $\min ^{-1}$ & 20,000 & 1 & 20,000 & 0.0 \\
\hline & $E_{2}$ & $\mathrm{~J} \mathrm{~mol}^{-1}$ & 67,500 & 35,000 & 675,000 & 0.0 \\
\hline & $a$ & & 0.40 & 0.10 & 0.40 & 0.0 \\
\hline & $b$ & & 0.30 & 0.10 & 0.30 & 0.0 \\
\hline \multirow[t]{6}{*}{0.001} & $A_{1}$ & $\min ^{-1}$ & $1.07 \times 10^{8}$ & $1.7 \times 10^{6}$ & $1.09 \times 10^{8}$ & 1.90 \\
\hline & $E_{1}$ & $\mathrm{~J} \mathrm{~mol}^{-1}$ & 101,000 & 70,000 & 101,092 & 0.09 \\
\hline & $A_{2}$ & $\min ^{-1}$ & 20,000 & 1 & 22,121 & 10.6 \\
\hline & $E_{2}$ & $\mathrm{~J} \mathrm{~mol}^{-1}$ & 67,500 & 35,000 & 68,101 & 0.89 \\
\hline & $a$ & & 0.40 & 0.10 & 0.399 & 0.01 \\
\hline & $b$ & & 0.30 & 0.10 & 0.299 & 0.02 \\
\hline
\end{tabular}

\section{Numerical Simulation}

The simulated measurements of total mass $M(t)$ are obtained from the solution of the direct problem, by using a priori prescribed values for the unknown parameters to be recovered simultaneously. The exact values of the parameters are reported in Table 1 . The other quantities are taken as $\beta=10 \mathrm{~K} \mathrm{~min}^{-1}, T_{\min }=450 \mathrm{~K}, T_{\max }=850 \mathrm{~K}$.

The solution of the direct problem, obtained using known parameters, provides the exact total mass measurements $M_{x}\left(t_{i}\right), i=1, \ldots, N$ (errorless). Measurements containing random errors are simulated by adding a white noise (error term) to $M_{x}\left(t_{i}\right)$ in the form:

$$
M\left(t_{i}\right)=M_{x}\left(t_{i}\right)+\omega \sigma
$$

where $M\left(t_{i}\right)$ is the simulated measurements of total mass, $M_{x}\left(t_{i}\right)$ is the exact total mass (errorless), $\sigma$ is the standard deviation of the measurement errors, and $\omega$ is a random variable with normal distribution, zero mean and unitary standard deviation. For the $99 \%$ confidence level we have $-2.576<\omega<+2.576$. This variable is generated with the subroutine DRRNOR of the IMSL library (IMSL, 1987). Figure 2 shows the solution of the direct problem using the exact parameters given in Table 1.

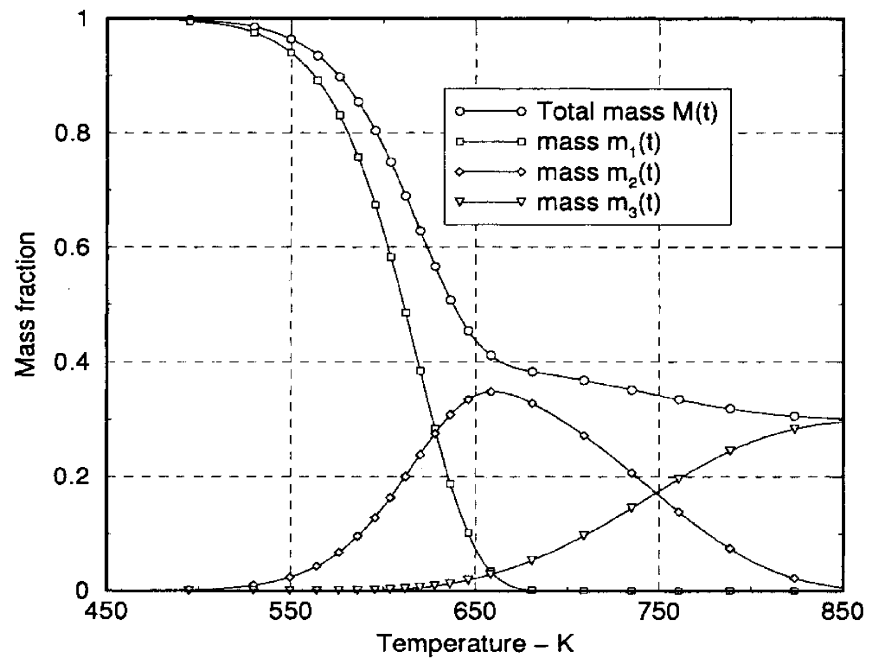

Figure 2. Solution of the direct problem with exact data $(\sigma=0)$ shown in Table 1.
We now consider the inverse problem of estimating simultaneously the components of the vector $\mathbf{U}$ by the Levenberg-Marquardt method. The initial guesses for the unknown parameters are displayed in Table 1. The computations were performed using a Pentium computer, under the Fortran PowerStation platform. The relative error of the estimated parameters is defined as

$$
\varepsilon_{i}=\left|\frac{u_{i}-\bar{u}_{i}}{\bar{u}_{i}}\right| \times 100 \% \quad i=1, \ldots, P
$$

where the overbar designates the exact parameter under hand.

Table 1 summarizes the results obtained for the simultaneously estimation of six parameters. It shows the initial guess of the unknowns, their recovered and exact values, and their estimation error, respectively. Two levels of measurement errors are considered for numerical analysis including $\sigma=0$ (errorless) and $\sigma=0.001$, which corresponds approximately to $0.1 \%$ of the maximum of total mass $M(t)$ during the pyrolysis process.

By using the errorless measurements $(\sigma=0)$, the six parameters are recovered exactly in $47 \mathrm{~s}$ of CPU time and the estimated loss weight matches precisely the measured one. We should mention here that, beyond the displayed initial guess, we cannot get convergence of the estimation algorithm. We observe that, except for the parameter $E_{2}$, the initial guess of the remain parameters is wide enough with respect to their final values (exact values).

Also, Table 1 shows the estimated parameters when they are obtained with measurements containing random errors according to equation (17). Generally, the estimated parameters are in good agreement with their exact values. The highest error is observed with parameter $A_{2}$ and this results from its low sensitivity as underlined in the sensitivity analysis section. The estimation error of parameter $A_{1}$ is relatively high with respect to the noise level added to the exact data in generating the simulated measurements. Also, this can be explained by its low sensitivity, as shown in Figure 1.

\section{Real Test Case}

\section{Pyrolysis of cardboard}

Experiments were performed using a SETERAM ATGDSC-111 apparatus. To generate dynamic data, different cycles were realized for which the temperature increased linearly with time and at different rates, $\beta=\mathrm{d} T / \mathrm{d} t$ $\left(\mathrm{K} \mathrm{min}^{-1}\right)$. The upper temperature value was chosen to ensure that pyrolysis process was complete, as mentioned in the sensitivity analysis.

Figure 3 shows the experimental and model predicted mass evolutions versus temperature obtained with the developed algorithm. Small differences can be observed around the curvature of the measured total loss weight and the fitting can be considered as acceptable and efficient. Also, Figure 3 displays the residual of the weight loss, i.e. $M(t)-Y(t)$. The biggest difference is observed over the temperature domain $[600,700]$ and the loss mass residual is still less than $2 \%$. The residual is scattered around zero line during the whole experimental temperature change. If the fitting was perfect, i.e. the fitting matches precisely the mean measured profile, we would get the measurement errors 


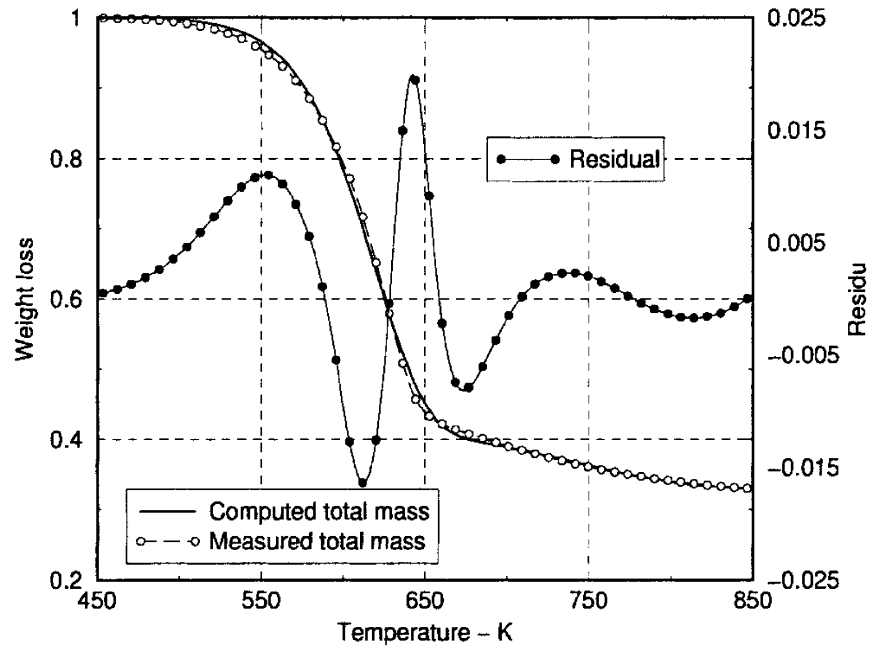

Figure 3. Comparison between the experimental and the computed loss mass evolution. Weight loss residual evolution as temperature function. The heating rate was taken as $\beta=10 \mathrm{~K} \mathrm{~min}^{-1}$.

(noise) instead of the model fluctuations. Figure 4 displays the computed total mass vs the total measured mass loss. As mentioned in the residual analysis, the estimation is acceptable since this figure shows approximately a straight line representing the bisection of the principal axis of Figure 4.

By using the experimental data of cardboard pyrolysis, the values of estimated parameters are: $A_{1}=1.07 \times$ $10^{8} \mathrm{~min}^{-1}, \quad E_{1}=101,107 \mathrm{~J} \mathrm{~mol}^{-1}, \quad A_{2}=19,824 \mathrm{~min}^{-1}$, $E_{2}=67,648 \mathrm{~J} \mathrm{~mol}^{-1}, a=0.41$ and $b=0.32$.

These parameters are obtained in less than $50 \mathrm{~s}$ CPU time and with the same initial guesses as displayed in Table 1. In comparison with the estimation procedure developed in our previous paper (David et al., 2003) around the function fmins of Matlab software (MATLAB, 1999), where the estimation time takes over $2 \mathrm{~h}$ of computing time, the presented method is more than 100 times faster. This tool will be used in the future to facilitate model building. Finally, the results shown were obtained with a personal computer powered by an Intel ${ }^{\circledR}$ Pentium 4 processor of $2 \mathrm{GHz}$, and using $256 \mathrm{MB}$ of RAM, under the Fortran Powerstation platform.

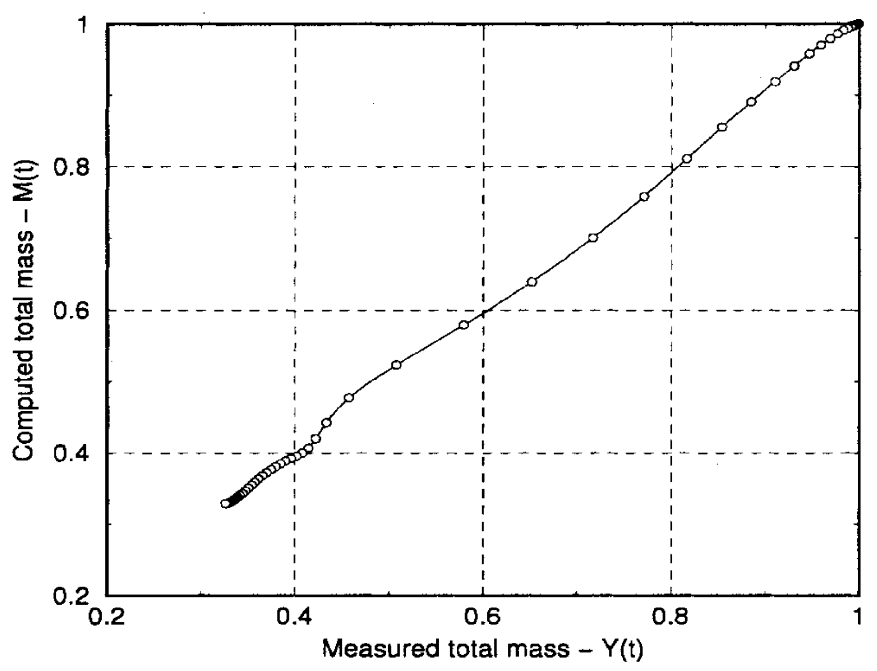

Figure 4. Computed total mass loss versus measured total mass loss.

\section{CONCLUDING REMARKS}

A numerical procedure is presented for the simultaneous estimation of kinetic parameters characterizing the pyrolysis process of cardboard. The minimization procedure is conducted by minimizing the square difference between experimental measured total mass and the corresponding calculated values from a mathematical model.

A comparison between recovered and exact data showed good agreement. The obtained results underline the robustness of the algorithm and its capabilities to recover simultaneously the kinetic parameters using less a priori information and wide deviation in the initial guess of parameters. This tool is developed by taking into consideration some physical constraints such as positiveness of kinetic parameters, and continuity of reaction schemes.

Despite a relatively wide deviation in the initial guess of parameters to be recovered, the presented tool is still sensitive to the initial values. This deficiency is due to the strong non-linearity of the kinetic reacting schemes. Efforts are currently underway to address this problem by considering the genetic algorithms in obtaining the best initial guess. Also, the direct estimation of the Arrhenius functions instead of Arrhenius parameters will be analysed by means of function estimation tools.

\section{NOMENCLATURE}

$\begin{array}{ll}A_{1}, A_{2} & \text { Arrhenius factor, } \mathrm{min}^{-1} \\ b & \text { constant } \\ c & \text { constant } \\ E_{1}, E_{2} & \text { activation energy, } \mathrm{J} \mathrm{mol}^{-1} \\ k_{1}, k_{2} & \text { kinetic reaction rate, } \mathrm{min}^{-1} \\ \mathbf{M} & \text { vector of computed mass } \\ M\left(t_{i}\right) & \text { computed total mass at time } t_{i} \\ m_{1}, m_{2}, m_{3} & \text { mass fraction } \\ N & \text { number of measurements } \\ P & \text { number of parameters } \\ R & \text { universal gas constant, } \mathrm{kJ} \mathrm{kmol}^{-1} \mathrm{~K}^{-1} \\ T & \text { temperature, } \mathrm{K} \\ t & \text { time, min } \\ \mathbf{U} & \text { vector of unknown parameters } \\ u_{i} & \text { unknown parameter } i \\ \mathbf{X} & \text { sensitivity matrix } \\ X_{i j} & \text { sensitivity coefficient } \\ X_{i j}^{+} & \text {relative sensitivity coefficient } \\ \mathbf{Y} & \text { vector of measured mass } \\ Y\left(t_{i}\right) & \text { measured mass at time } t_{i} \\ G r e e k \text { symbols } & \\ \beta & \\ \varepsilon & \text { heating rate } \\ \mu & \text { small number } \\ \mathbf{\Omega} & \text { damping parameter } \\ & \text { damping matrix } \\ & \end{array}$

\section{REFERENCES}

Bard, Y., 1974, Nonlinear Parameter Estimation (Academic Press, New York, USA).

Beck, J. and Arnold, K., 1977, Parameter Estimation in Engineering and Science (Wiley Interscience, New York, USA).

David, C., Salvador, S., Dirion, J. and Quintard, M., 2003, Determination of a reaction scheme for cardboard thermal degradation using thermal gravimetric analysis, J Anal Appl Pyrol, 67(2): 307-323.

Font, R., Martin-Gullon, I., Esperanza, M. and Fullan, A., 2001, Kinetic law for solids decomposition. Application to thermal degradation of heterogeneous materials, J Anal Appl Pyrol, 58-59: 703-731.

IMSL, 1987, Library Edition 10.0, User's Manual, Math/Library (IMSL Houston, TX, USA). 
Levenberg, K., 1944, A method for the solution of certain non-linear problems in least square, $Q$ Appl Math, 2(2): 164-168.

Marquardt, D.W., 1963, An algorithm for least square estimation of nonlinear parameters, J Soc Ind Appl Math, 11(2): 431-441.

MATLAB, 1999, Library Edition 5.3, User's Manual (MathWorks Inc., Natick, MA, USA).

More, J., 1978, Numerical Analysis, Lectures Notes in Mathematics, Dold, E.B. and Watson, G. (eds), Vol. 630 (Springer, Berlin, Germany), pp 105116.

Opfermann, J., 2000, Kinetic analysis using multivariate non-linear regression. (i) Basic concepts, J Therm Anal Calc, 60: 641-658.

Press, W., Teukolsky, S., Vetterling, W. and Flannery, B., 1992, Numerical Recipes in Fortran (Cambridge University Press, Cambridge, UK).

Roduit, M.M.B. and Baiker, A., 1996, Influence of experimental conditions on the kinetic parameters of gas-solid reactionsparametric sensitivity of thermal analysis, Thermochim Acta, 282/283: 101-119.

\section{ACKNOWLEDGMENT}

The authors would like to express their deep acknowledgements to the reviewers for their constructive critic and remarks in improving the quality of this paper.

\section{ADDRESS}

Correspondence concerning this paper should be addressed to $\mathrm{Dr} \mathrm{T}$. Loulou, Ecole des Mines d'Albi-Carmaux, Laboratoire de Gnie des Procds des Solides diviss, UMR CNRS 2393, 8100 Albi, France.

E-mail: loulou@enstimac.fr 